\title{
Multiple Transceivers Inter-satellite Optical wireless communication System Performance
}

\author{
Abd El-Naser Mohamed \\ Electronics and Electrical \\ Communications Engineering \\ Department, Faculty of Electronic \\ Engineering, Menoufia University, \\ Egypt.
}

\author{
Ahmed Rashed \\ Electronics and Electrical \\ Communications Engineering \\ Department, Faculty of Electronic \\ Engineering, Menoufia University, \\ Egypt. \\ Ehab. S. Hashim \\ Electronics and Electrical \\ Communications Engineering \\ Department, Faculty of Electronic \\ Engineering, Menoufia University, \\ Egypt.
}

\author{
Eman El-gammal \\ Electronics and Electrical \\ Communications Engineering \\ Department, Faculty of Electronic \\ Engineering, Menoufia University, \\ Egypt.
}

\begin{abstract}
This Study clarifies the effect of using multi transceiver channel in the inter-satellite optical wireless communication system. The results obtained in this research have been simulated by optisystem version 7. This study clarifies the impact of applying 16 transceiver on the system performance. Also, it represents comparison between inter-satellite optical wireless communication system using single channel and the other used 16 transceiver at different wavelengths. Then, it clarifies the impact of increasing transmission data rate on the system performance at different propagation distances for 16 transceiver system. The impact of transmitted power on the system performance also has been explained. The performance parameters in our study are quality factor and received power.
\end{abstract}

\section{Introduction}

Optical wireless communication (OWC) is one of the most leading implementation depends on laser. OWC has many advantages over than radio frequency link such as its ability to send high speed data rate to thousands of kilometers with small pay load and that reduce the cost. The wavelength of RF is longer than it in laser so the beam width result from laser is narrower than beam width result from laser. Loss result from OWC is small compared to loss in case of RF link. In IsOWC communication occurs between satellite and another whether in the same orbit or in different orbit [1-4].

Satellite has been categorized into three orbits. The first orbit is low earth orbit (LEO), which ranges from 1000 to $5000 \mathrm{~km}$ above the earth, it takes approximately 2 hours for a full orbit. Due to the proximity to Earth, LEO satellites request less amplification for transmission. The second orbit is medium earth orbit (MEO) which is existing between LEO and GEO satellites and its altitude to the earth ranges from 5000 to $20000 \mathrm{~km}$.
It takes approximately 4 hours for a full orbit. This satellite is used for shipping systems and also used for voice and data communications. The third orbit is geostationary earth orbit (GEO) which its altitude to the earth is $36000 \mathrm{~km}$. It takes approximately 24 hours for a full orbit. In IsOWC data signal must be encoding because signal encoding has many advantages such as saving bandwidth, and error correction. Also encoding data protect it from visibility to any one not sharing on the network [5-8]. In IsOWC systems light signal in satellite transmitter is modulated according to signal produced by pulse generator and transmitted through optical wireless channel to another satellite receiver. There are different methods and techniques can be used in IsOWC to improve the system performance [9-13]. In this paper, transmission between two satellites is in low earth orbit (LEO) and the medium is considered a vacuum. There are many parameters affect the system performance such as wavelength, bit rate and distance. By increasing wavelength, bit rate and distance the system performance decreases. By increasing number of transceiver, the system performance improves.

\section{Simulation of 16 transceiver IsOWC System Model}

OWC consists of transmitter, channel which represents transmission medium and receiver. This model consists of 16 transceiver. Transmitter consists of pseudo random bit sequence generator, which generate the data signal. The generated data signal has been encoded by NRZ pulse generated. Then optical signal generated from light source is modulated by $\mathrm{MZ}$ modulator according to encoded signal out from NRZ pulse generated. The optical modulated signal has been splitted to 16 signal using splitter. Power combiner used to combine the splitted signals from each splitter and send them over OWC channels which represent the propagating medium for transmitted signals. The 16 received optical signals have 
combined to one signal by power combiner and then, the combined received signal has converted to electrical signal by avalanche photo diode. Then, electrical signal is filtered by using low pass Bessel filter. The 3R regenerator uses for retiming, regeneration, reshaping.

\section{Simulation Results and Discussions}

The operating parameters used in this paper are the following, operating wavelength is $1550 \mathrm{~nm}$, antenna diameter $25 \mathrm{~cm}$ and transmitted power is $12 \mathrm{dBm}$ [1], and propagation distance ranges from 1000 to $5000 \mathrm{~km}$. Vacuum is assumed to be the medium between transmitter and receiver. Propagation has occurred in line of sight. Low pass Bessel filter has frequency equal $0.75 \mathrm{x}$ bit rate and its order is 4 . This paper studies the impact of applying 16 transceiver on the system performance. The quality factor is a parameter that describes the resonance behavior of the system.

Section 1 represents the comparison between system performance of $16 \mathrm{TX} / \mathrm{RX}$ IsOWC system and simple IsOWC system [1] at propagation distance ranges from $500 \mathrm{~km}$ to $2500 \mathrm{~km}$ and transmission data rate is $80 \mathrm{Mb} / \mathrm{s}$ [1]. Section 2 represents the system performance of simple and $16 \mathrm{TX} / \mathrm{RX}$ IsOWC system at wavelengths 850 $\mathrm{nm}, 950 \mathrm{~nm}, 1310 \mathrm{~nm}$ and $1550 \mathrm{~nm}$, transmission data rate is $80 \mathrm{Mb} / \mathrm{s}$, transmitted power is $12 \mathrm{dBm}$ and propagation distance is $1000 \mathrm{~km}$. Section 3 represents the relation between quality factor and transmission data rate at different propagation distances. Section 4 represents the impact of transmitted power on system performance at propagation distance $5000 \mathrm{~km}$.

\subsection{Comparison between system performance for 16 TX/RX IsOWC system and simple IsOWCsystem[1].}

Fig. 2 shows the relation between received power and propagation distance for Simple IsOWC system and 16 TX/RX IsOWCsystem. It is observed that by increasing propagation distance, the received power decreases.The system performance improved by applying $16 \mathrm{TX} / \mathrm{RX}$ on the system. For example, the received power at distance $1000 \mathrm{~km}$ for proposed model is $-23.6 \mathrm{dBmbut}$ in case of reference model [1] is -59.6 . So the obtained received power in case of applying $16 \mathrm{TX} / \mathrm{RX}$ is better than it in simple model [1]. The results in fig. 1 is shown in Table 1 .

\subsection{The system performance of $16 \mathrm{TX} / \mathrm{RX}$ and simple IsOWC system at different values of wavelengths.}

Figures (3-6) show the eye diagram of the IsOWC system for one transceiver and $16 \mathrm{TX} / \mathrm{RX}$ at different wavelengths. It is observed that by increasing wavelength the system performance decreases. The system performance at wavelength $850 \mathrm{~nm}$ in both systems is better than system performance at $950 \mathrm{~nm}, 1310 \mathrm{~nm}$ and $1550 \mathrm{~nm}$. It is shown that the quality factor for $16 \mathrm{TX} / \mathrm{RX}$ system at wavelength $850 \mathrm{~nm}$ is 6396.5 quality factor at wavelength $1550 \mathrm{~nm}$ is 3798.4 . The system performance for $16 \mathrm{TX} / \mathrm{RX}$ system at all wavelengths is better than it for simple model system [1]. For example in fig. 3.the quality factor for $16 \mathrm{TX} / \mathrm{RX}$ at wavelength $850 \mathrm{~nm}$ is 6396.5 and quality factor for simple model [1] is 562.6. The difference between two cases is too large and that illustrated in table. 2 .

\subsection{Relation between signal quality and transmission data rate at different distances.}

Fig. 7 shows the relation between quality factor and transmission data rate at different values of propagation distances ranges from $1000 \mathrm{~km}$ to $5000 \mathrm{~km}$ and wavelength is $1550 \mathrm{~nm}$. It is observed that increasing transmission data rate leads to decreasing quality factor. Transmission data rate ranges from $20 \mathrm{~Gb} / \mathrm{s}$ to $100 \mathrm{~Gb} / \mathrm{s}$. The best system performance is obtained at propagation distance $1000 \mathrm{~km}$. The least quality factor has obtained for all used transmission data rate is at propagation distance $5000 \mathrm{~km}$. Increasing propagation distance leads to decreasing. Table. 3 shows the results which has simulated at fig. 7 .

\subsection{Relation between signal quality and transmitted power.}

Fig. 8 clarifies the impact of increasing transmitted power on the system performance at propagation distance 5000 $\mathrm{km}$ and transmission data rate $200 \mathrm{~Gb} / \mathrm{s}$. The transmitted power is chosen in range from $10 \mathrm{dBm}$ to $25 \mathrm{dBm}$. By increasing transmitted power, quality factor increases. At transmitted power $25 \mathrm{dBm}$ the quality factor 68.3 . If the transmission data rate in this case increases than $200 \mathrm{~Gb} / \mathrm{s}$, the quality factor at transmitted power $10 \mathrm{dBm}$ will be very small and the signal will be very bad. The results plotted in fig. 8 is shown in table. 4 . At transmitted power $25 \mathrm{dBm}$ and propagation distance $5000 \mathrm{~km}$, the used transmission data rate can be reached to $600 \mathrm{~Gb} / \mathrm{s}$ and the quality factor in this case is 39.4. Also at transmitted power $25 \mathrm{dBm}$ and transmission data rate $200 \mathrm{~Gb} / \mathrm{s}$ the propagation distance can be extended to $20000 \mathrm{~km}$ at the same previous operating parameters and the quality factor in this case is 10.1 .

Table 1.Comparison between received power for reference model [1] and proposed model

\begin{tabular}{|c|c|c|}
\hline \multirow{2}{*}{$\begin{array}{c}\text { Distance } \\
(\mathrm{km})\end{array}$} & \multicolumn{2}{|c|}{$\begin{array}{c}\text { Received Power, } \mathrm{P}_{\mathrm{R}} \\
(\mathrm{dBm})\end{array}$} \\
\cline { 2 - 3 } & $\begin{array}{c}\text { Reference } \\
\text { model }\end{array}$ & $\begin{array}{c}\text { Proposed } \\
\text { model }\end{array}$ \\
\hline 500 & -47.6 & -11.5 \\
\hline 1000 & -59.6 & -23.6 \\
\hline 1500 & -66.7 & -30.6 \\
\hline 2000 & -71.6 & -35.6 \\
\hline 2500 & -75.4 & -39.5 \\
\hline
\end{tabular}




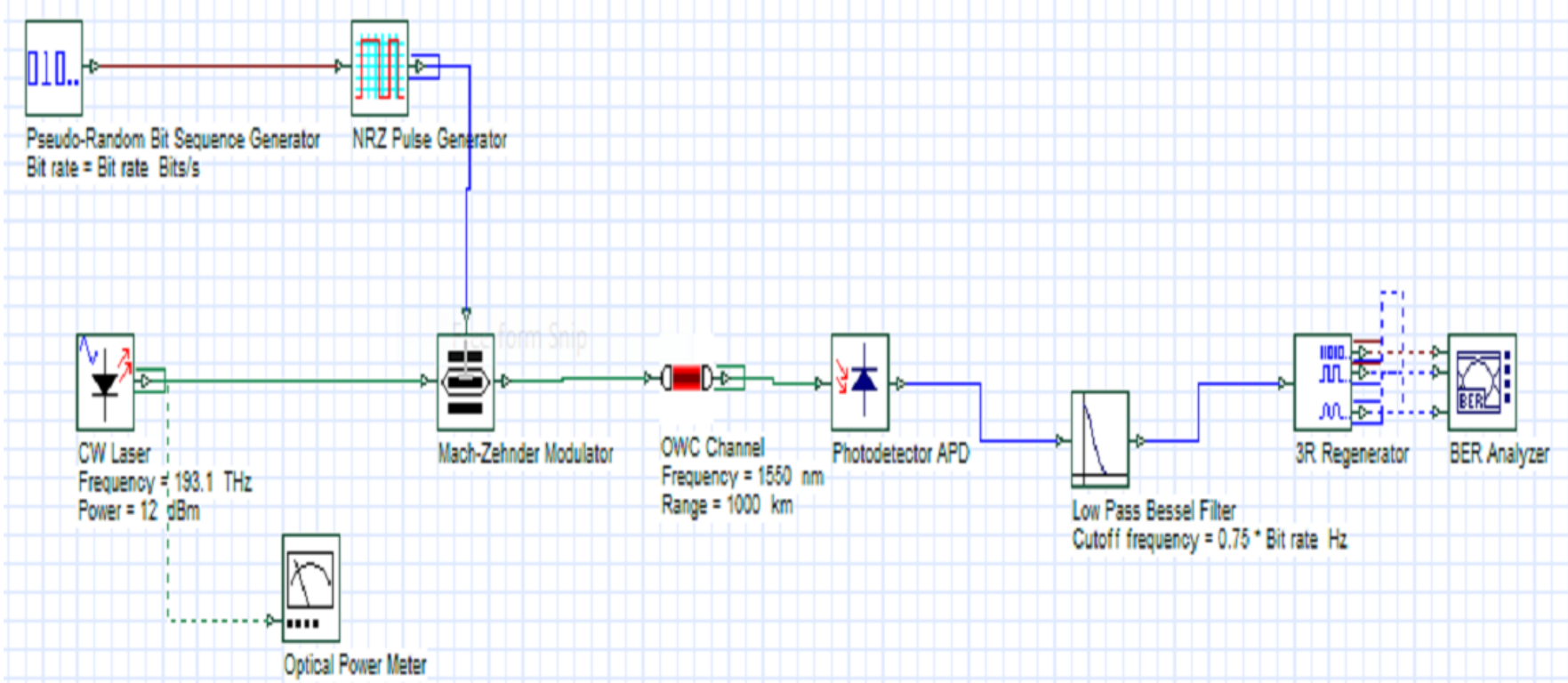

Fig. 1. A. Simple model of IsOWC system.

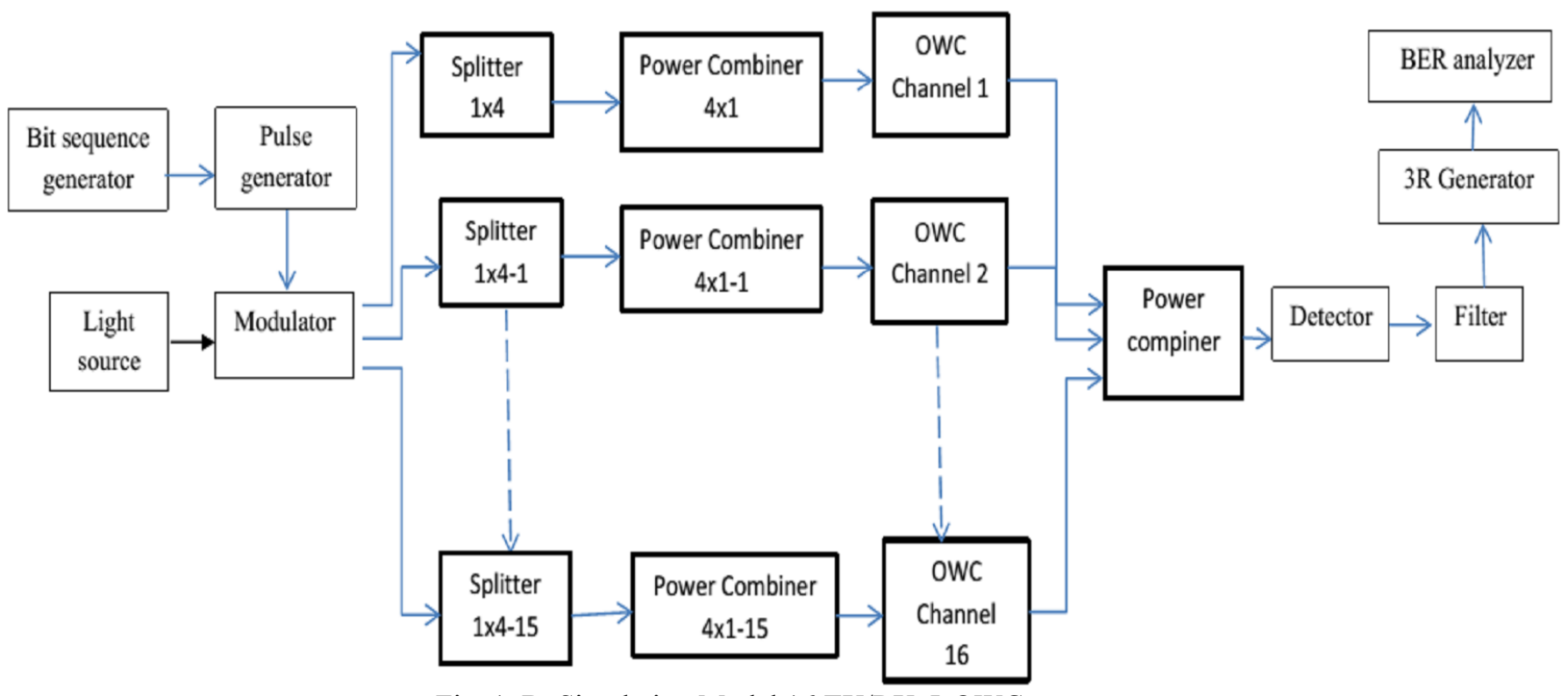

Fig. 1. B. Simulation Model 16 TX/RX IsOWC system.

Propagation distance $(\mathrm{km})$

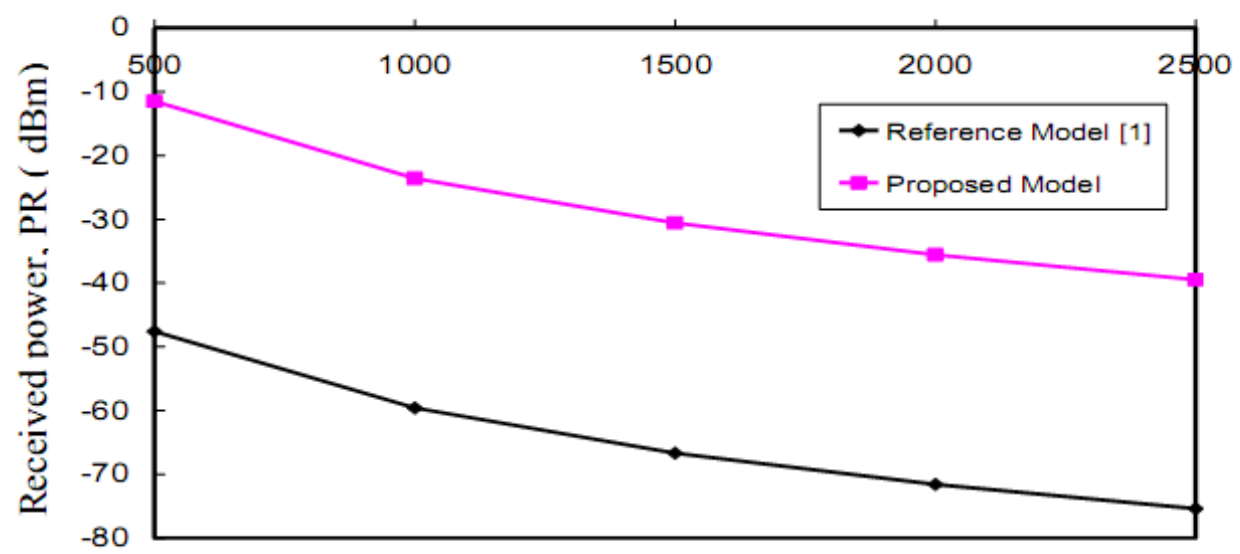

Fig. 2. Received power in relation to propagation distance using reference model [1] and proposed model.

Fig. 2 Received power in relation to propagation distance using reference model [1] and proposed model. 

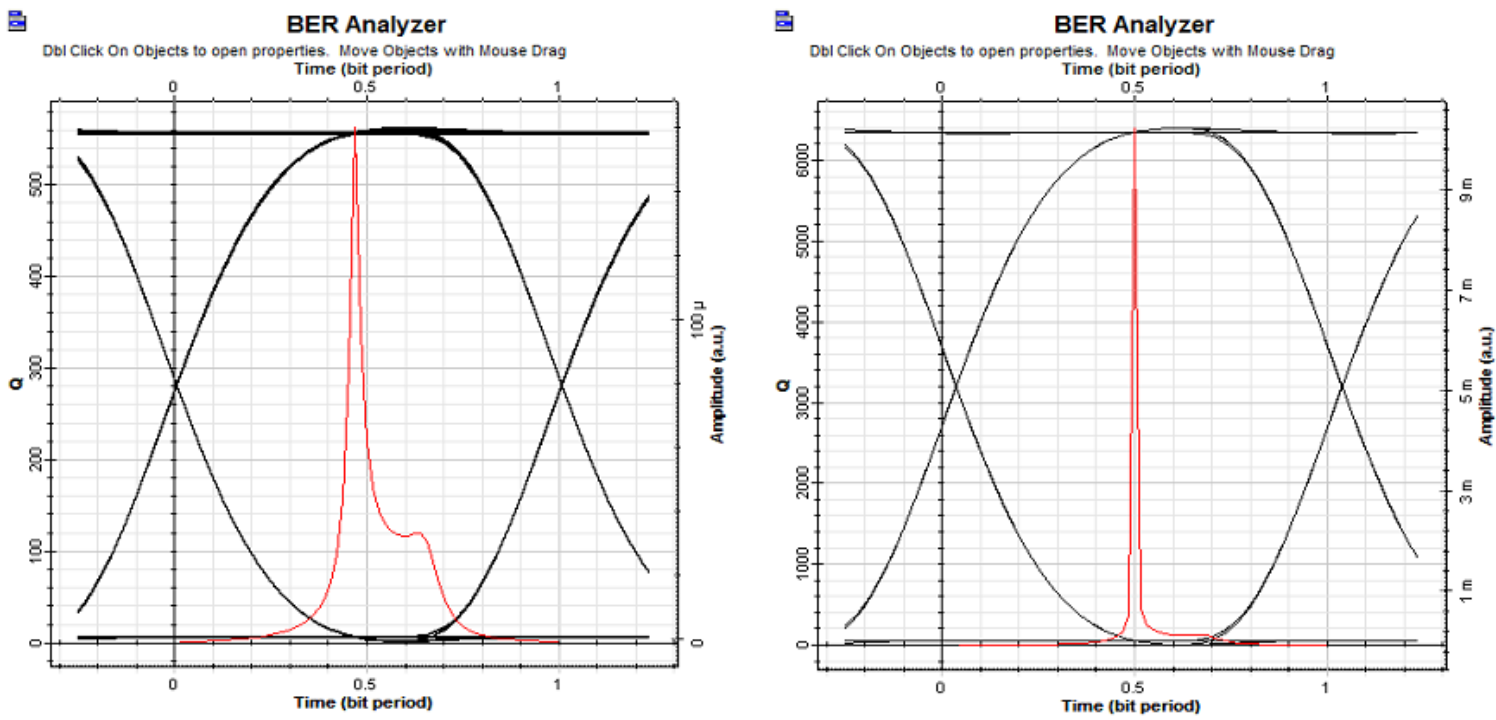

(a) simple IsOWC system [1] (b) $16 \mathrm{TX} / \mathrm{RX}$ IsOWC system

Fig. 3. Eye diagram of the simple and $16 \mathrm{Tx} / \mathrm{RX}$ IsOWC at wavelength $850 \mathrm{~nm}$.
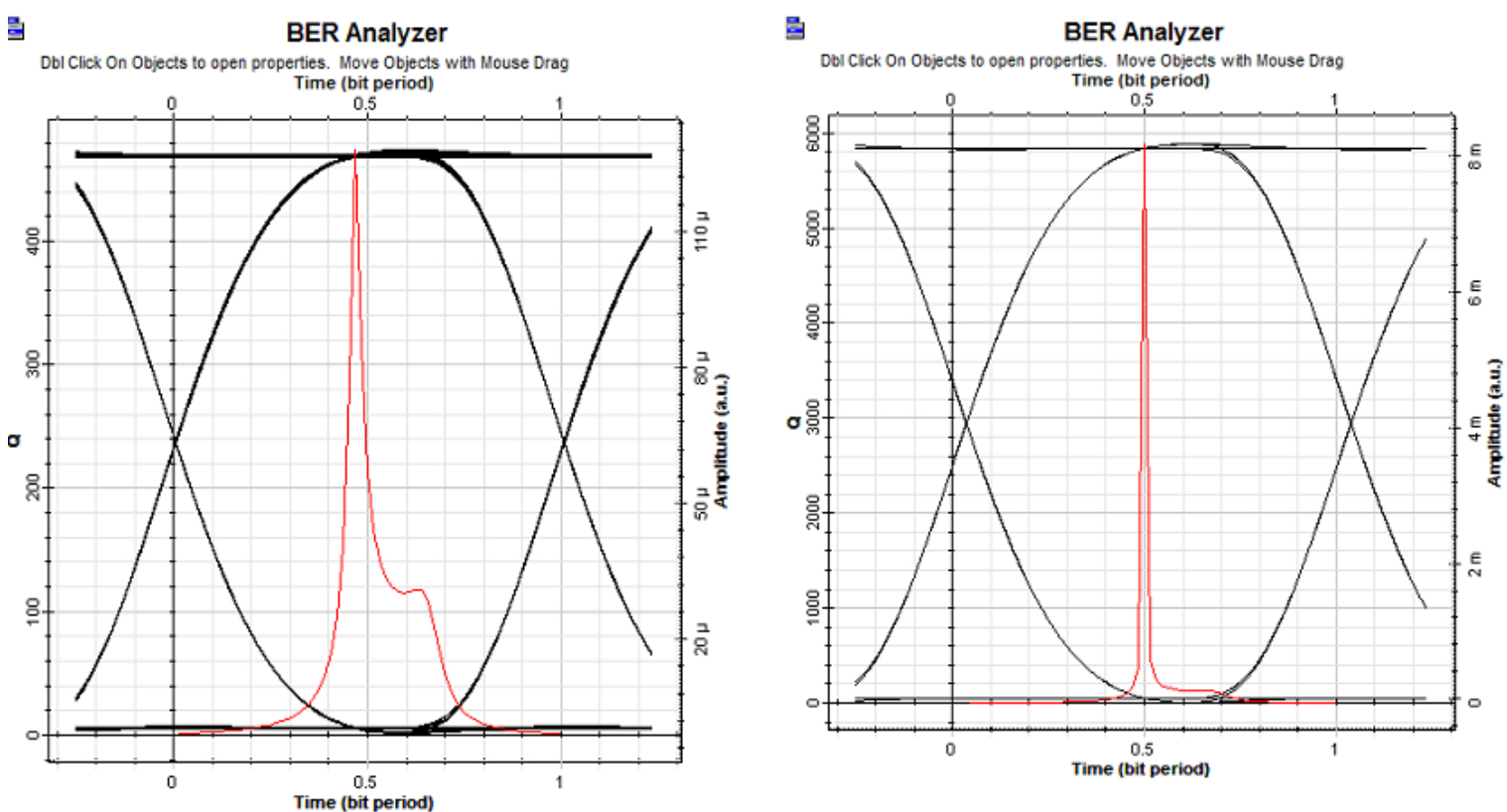

(c) simple IsOWC system [1] (d) $16 \mathrm{TX} / \mathrm{RX}$ IsOWC system

Fig. 4. Eye diagram of the simple and $16 \mathrm{Tx} / \mathrm{RX}$ IsOWC at wavelength $950 \mathrm{~nm}$.
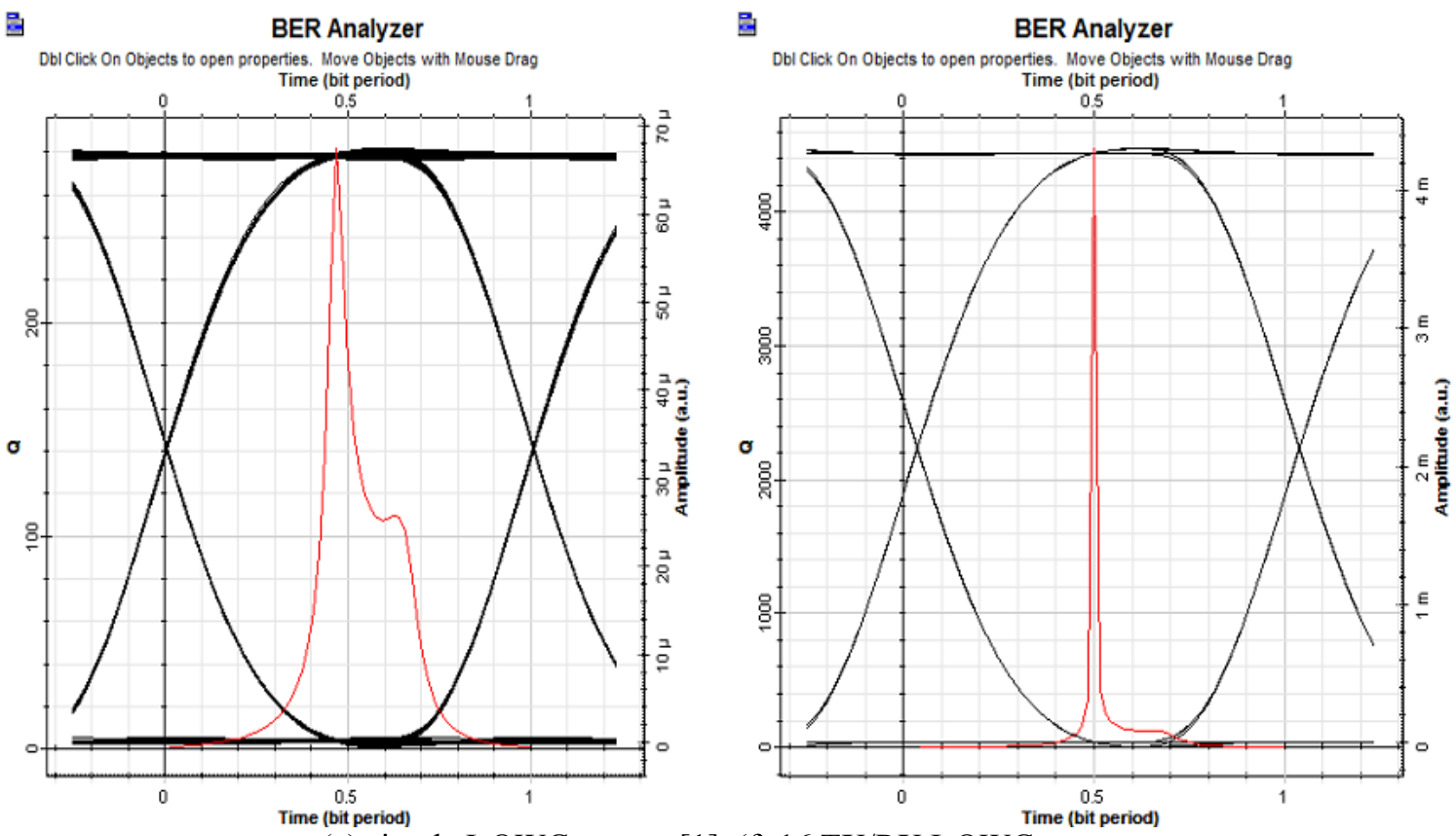

(e) simple IsOWC system [1] (f) $16 \mathrm{TX} / \mathrm{RX}$ IsOWC system

Fig. 5. Eye diagram of the simple and $16 \mathrm{Tx} / \mathrm{RX}$ IsOWC at wavelength $1310 \mathrm{~nm}$. 


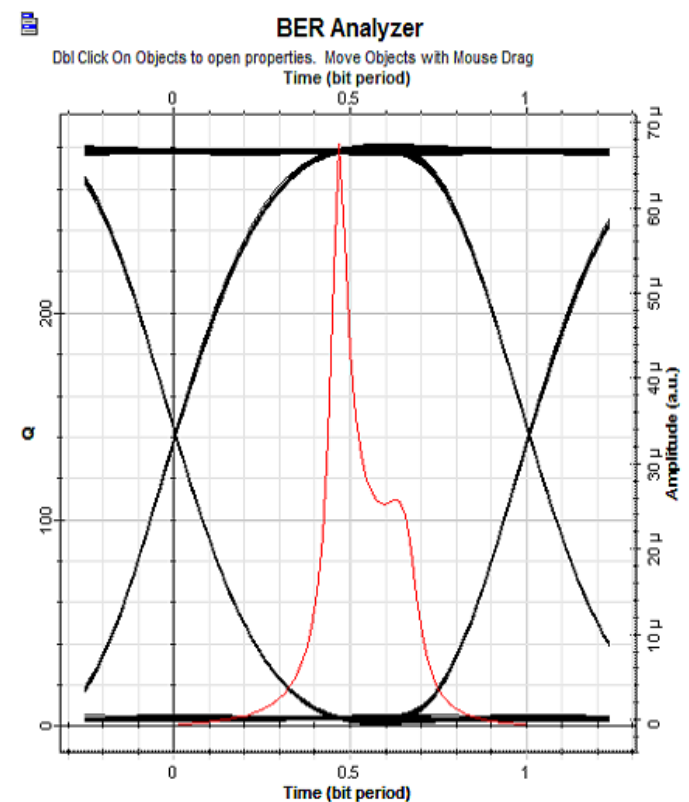

(g) simple IsOWC system [1]

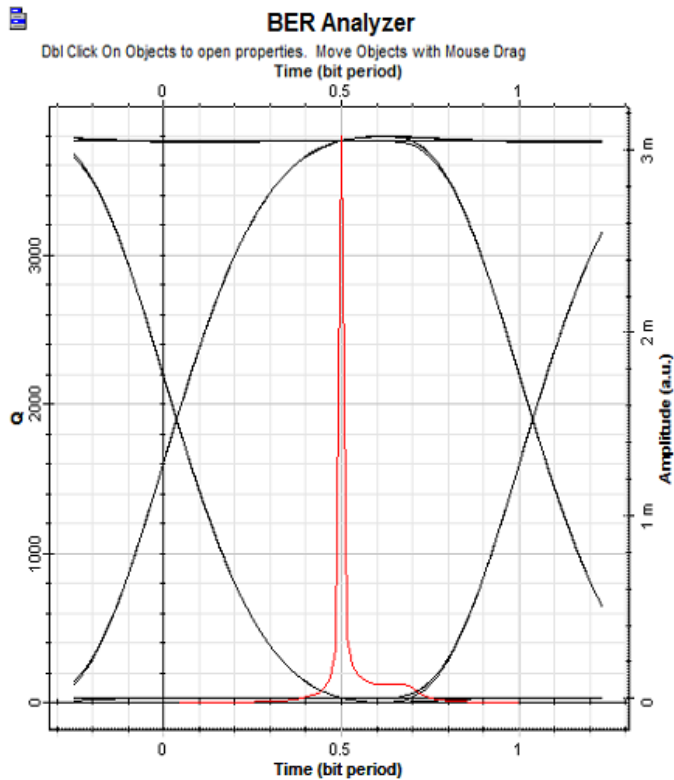

(h) $16 \mathrm{TX} / \mathrm{RX}$ IsOWC system

Fig. 6. Eye diagram of the simple and $16 \mathrm{Tx} / \mathrm{RX}$ IsOWC at wavelength $1550 \mathrm{~nm}$

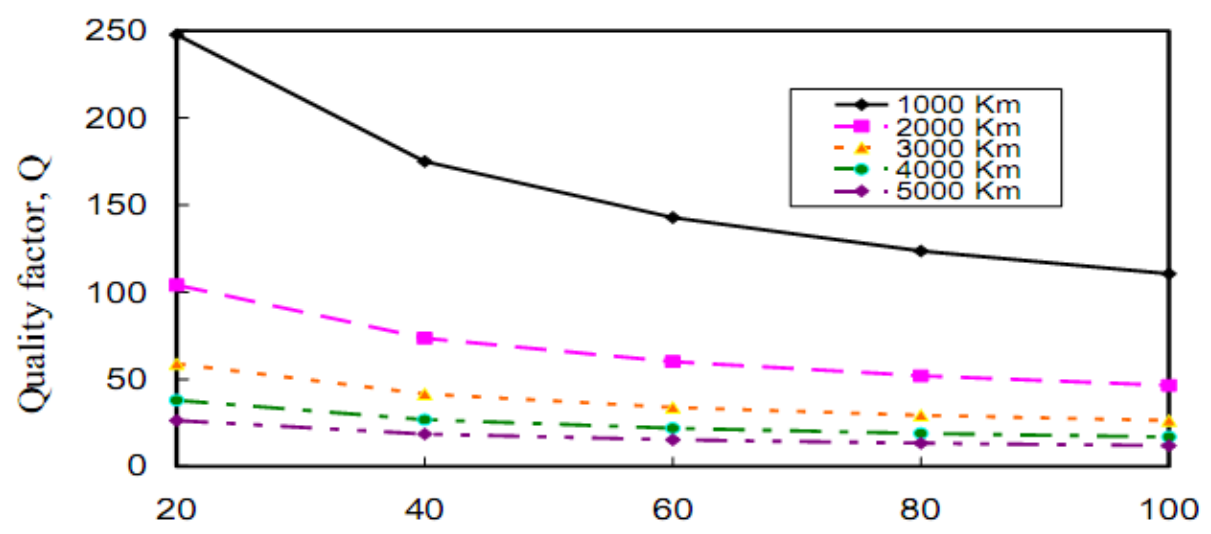

Transmission data rate $(\mathrm{Gb} / \mathrm{s})$

Fig. 7. Signal quality factor in relation to transmission data rate at different values of propagation distance.

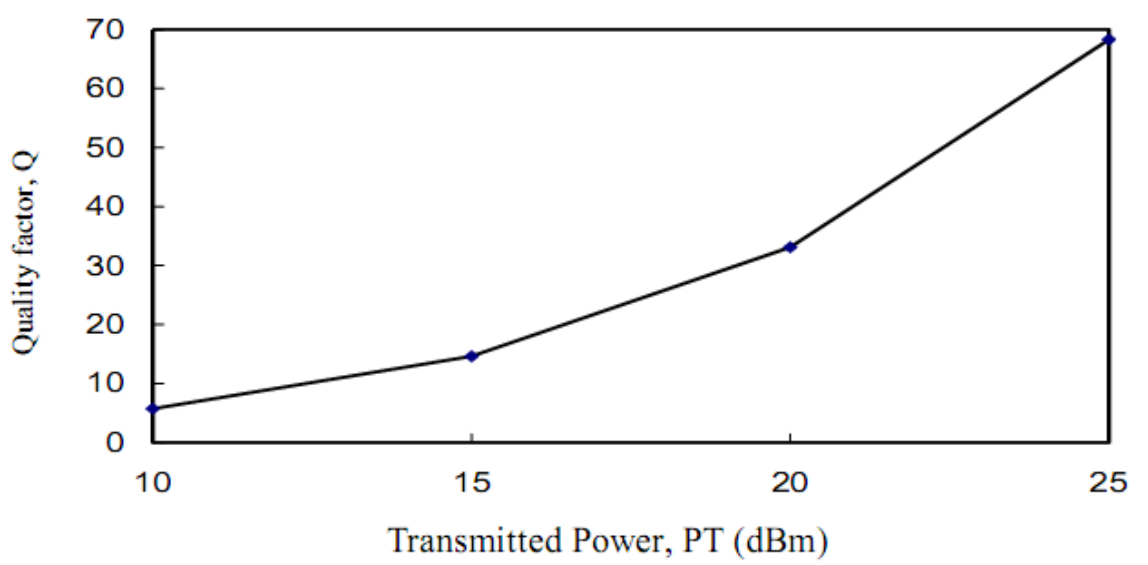

Fig. 8. Signal quality factor in relation transmitted power.

Table 3.Relation between quality factor and transmission data rate at different values of distances.

\begin{tabular}{|c|c|c|c|c|c|}
\hline \multirow{2}{*}{$\begin{array}{c}\text { Distance } \\
(\mathrm{km})\end{array}$} & \multicolumn{5}{|c|}{ Quality factor, Q } \\
\cline { 2 - 6 } & $20 \mathrm{~Gb} / \mathrm{s}$ & $40 \mathrm{~Gb} / \mathrm{s}$ & $60 \mathrm{~Gb} / \mathrm{s}$ & $80 \mathrm{~Gb} / \mathrm{s}$ & $100 \mathrm{~Gb} / \mathrm{s}$ \\
\hline 1000 & 247.8 & 175 & 142.8 & 123.6 & 110.6 \\
\hline 2000 & 104 & 73.6 & 60.1 & 52 & 46.5 \\
\hline 3000 & 58.9 & 41.6 & 33.9 & 29.4 & 26.3 \\
\hline 4000 & 37.9 & 26.8 & 21.9 & 18.9 & 16.9 \\
\hline 5000 & 26.3 & 18.6 & 15.3 & 13.3 & 11.9 \\
\hline
\end{tabular}


Table 2.Quality factor for simple model [1] and proposed model at different values of wavelengths.

\begin{tabular}{|c|c|c|}
\hline \multirow{2}{*}{$\begin{array}{c}\text { Wavelength } \\
(\mathrm{nm})\end{array}$} & \multicolumn{2}{|c|}{ Quality factor, Q } \\
\cline { 2 - 3 } & $\begin{array}{c}\text { Reference } \\
\text { model }\end{array}$ & $\begin{array}{c}\text { Proposed } \\
\text { model }\end{array}$ \\
\hline 850 & 562.6 & 6396.5 \\
\hline 950 & 474.3 & 5882.9 \\
\hline 1310 & 281.8 & 4475.1 \\
\hline 1550 & 211.2 & 3798.4 \\
\hline
\end{tabular}

Table 4. Relation between quality factor and transmitted power at transmission data rate $200 \mathrm{~Gb} / \mathrm{s}$.

\begin{tabular}{|c|c|}
\hline $\begin{array}{c}\text { Transmitted } \\
\text { power } \\
(\mathrm{dBm})\end{array}$ & $\begin{array}{c}\text { Quality } \\
\text { factor, } \\
\mathrm{Q}\end{array}$ \\
\hline 10 & 5.7 \\
\hline 15 & 14.6 \\
\hline 20 & 33.1 \\
\hline 25 & 68.3 \\
\hline
\end{tabular}

\section{Conclusion}

In a summary, applying multiple transceivers in IsOWC system improves the system performance. By increasing Wavelength, the quality signal decreases. The system performance for $16 \mathrm{TX} / \mathrm{RX}$ system at all wavelengths is better than it for simple model system [1].Increasing transmission data rate leads to decreasing quality factor. By increasing transmitted power, quality factor increases. If the transmission data rate in case of using $5000 \mathrm{~km}$ and wavelength $1550 \mathrm{~nm}$ increases than $200 \mathrm{~Gb} / \mathrm{s}$, the quality factor at transmitted power $10 \mathrm{dBm}$ will be very small and the signal will be very bad. At transmitted power $25 \mathrm{dBm}$ and propagation distance $5000 \mathrm{~km}$, the used transmission data rate can be reached to $600 \mathrm{~Gb} / \mathrm{s}$ and the quality factor in this case is 39.4. Also at transmitted power $25 \mathrm{dBm}$ and transmission data rate $200 \mathrm{~Gb} / \mathrm{s}$ the propagation distance can be extended to $20000 \mathrm{~km}$ at the same previous operating parameters and the quality factor in this case is 10.1 .

\section{References}

[1] R.R.S, R.M.J, " Performance Evaluation of Optical Intersatellite Links with Varied Parameters," International Journal of Engineering Research \& technology science, vol. 4, no. 1, pp. 271-273, 2015.

[2] N. Kaur, G. Soni, "Performance analysis of intersatellite optical wireless communication (IsOWC) system by using NRZ and RZ modulation," International Journal of Scientific and Research Puplication, vol. 5, no. 1, pp. 1-5, 2015.

[3] A. Z. M, H.A.Fayed, A.A.El Aziz and M.H.Aly, 'The Influence of Varying the Optical Wavelength on ISL Performance Recognizing High Transmission data rates," IOSR Journal of Electronics and Communication Engineering, vol. 9, no. 1, pp. 6470, 2014.

[4] N. Noor, A. W. Naji, W. Al-Khateeb, "Theoretical Analysis of Multiple Transmitters and Receivers on
The Performance of Free Space Optics link," IEEE International Conference on Space Science and Communication, pp. 291-295, 2011.

[5] F. A. Wahab, T. K. Leong, H. Zulkifili, M. I. B. Ibrahim, M. A. B. Talib, N. A. Zamri, O. K. Ibrahim, "Multiple Transmitters \& Receivers For Free Space Optical Communication Link Performance Analysis," Journal of Telecommunication, Electronic and Computer Engineering, vol. 8, no. 5, pp. 29-32, 2016.

[6] B. Patnaik, P. k. Sahu, "Inter-satellite Optical Wireless Communication System Design and Simulation," Institute of Engineering and Technology (IET) Communications, vol. 6, no. 16, pp. 2561-2567, doi:10.1049/iet-com.2012.0044, 2012.

[7] T. Mehmood, N. Hameed, "Modeling and Performance Analysis of 10 Gbps Inter-SatelliteLink (ISL) Inter-Satelitte Optical Wireless Communication (IsOWC) System between LEO and GEO Satelitte," IEEE $17^{\text {Th }}$ International Multi-Topic Conference, 2014.

[8] V. K. K. Sarath, V. Kumar, A. k. Turuk, S. K. Das, "Performance Analysis of Inter-Satellite Optical Wireless Communication" I.J. Computer Network and Information Security, vol. 4, pp. 22-28, 2017,doi: 10.5815 /ijenis.

[9] Y. Singh, "Performance Analysis of Optical Wireless Communication Channel Link at Various Transmission data rates," International Journal of Computer Science \& Engineering Technology (IJCSET), vol. 5, no. 1, pp. 26-30, 2014.

[10]H. Kaur, "Review on Inter-satellite Optical Wireless Communication System," International Journal of Advanced Research in Computer Science, vol. 8, no. 4, pp. 48-51, May 2017.

[11] K. Singh and M. S. Bhamrah, "Investigations of Transmitted Power in Inter-satellite Optical Wireless Communication," International Journal of Computer Sience and Information Technology \& Security, vol. 2, no. 3, pp. 568-573, 2012.

[12] H. A. Sharsher and E. M. Elgammal, "Operation Performance Evaluation of Inter-satellite Optical Wireless Communication Systems in Low Earth Orbits", International Journal of Advanced Research in Electronics and Communication Engineering, vol. 4, no. 5, pp. 1691-1698, 2015.

[13] S. Singh, G. Singh, R. Kaur and M. Singh, "Intersatellite Optical Wireless Communication System Design a Study," Trends in Opto-Electro \& Optical Communicatio, vol. 8, no. 1, pp. 20-24, 2018. 